\title{
PAPER
}

\section{The prevalence of frontal variant frontotemporal dementia and the frontal lobe syndrome in a population based sample of 85 year olds}

\author{
T B Gislason, M Siögren, L Larsson, I Skoog
}

See end of article for authors' affiliations

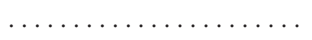

Correspondence to: Dr T B Gislason, The Institute of Clinical

Neuroscience, Department of Psychiatry, Sahlgrenska Hospital, S413 45

Gothenburg, Sweden thorstgi@landspitali.is

\section{Received}

19 February 2002

In revised form

30 January 2003

Accepted

17 February 2003

\begin{abstract}
Objectives: To investigate the prevalence of the frontal lobe syndrome (FLS) and the frontal variant of frontotemporal dementia (fvFTD) in a population based sample of 85 year olds.

Methods: A representative sample of 85 year olds $(n=451)$ in Gothenburg, Sweden was examined with a neuropsychiatric examination and a key informant interview performed by an experienced psychiatrist. A subsample underwent computed tomography (CT) of the head. The Lund-Manchester research criteria were used as a basis for a symptom algorithm to identify individuals with FLS and fvFTD. These were diagnosed blindly to the diagnosis of dementia according to DSM-III-R.

Results: A total of 86 individuals (19\%) fulfilled the criteria for FLS, and 14 of them fulfilled criteria for fvFTD. There were no differences between men and women. Among those with FLS, 75 (87\%) fulfilled DSM-III-R criteria for other types of dementia, mainly Alzheimer's disease and vascular dementia. Among the 14 fvFTD cases, only five were demented according to DSM-III-R. Moderate to severe frontal atrophy was found in $93 \%$ of those with FLS (and in all cases with fvFTD), but also in $49 \%$ of those without FLS. FLS was found in 35\% of those with moderate to severe frontal atrophy, and in $3 \%$ of those without these changes.

Conclusions: The prevalence of fvFTD was $3 \%$ in 85 year olds, which is higher than previously expected in this age group. Only a minority of those with fvFTD were detected by the DSM-III-R criteria for dementia. FLS was even more common, especially in those diagnosed with a dementia disorder.
\end{abstract}

$\mathrm{F}$ rontotemporal dementia (FTD) is a primary degenerative dementia with severe atrophy in the frontal and anterior temporal lobes. The current classification, as suggested by Hodges and Miller, categorises FTD as a general clinical label with three major subtypes. ${ }^{1}$ The most common subtype in clinical practice is frontal variant FTD (fvFTD), where the core symptoms are progressive changes in personality and behaviour with emotional and motivational blunting, and impairment in personal and social conduct. The other major subtypes are semantic dementia and progressive non-fluent aphasia, which present with a progressive disturbance in the expression of language. As a rule in FTD, memory loss is mild in the early stages, and patients are often oriented in their local environment. To facilitate an early and accurate diagnosis of FTD, a consensus report on the core clinical features of FTD was published in 1994. ${ }^{2}$ This report was revised in 1998 and an updated term, frontotemporal lobar dementia, was suggested. ${ }^{3}$ The Work Group on Frontotemporal Dementia and Pick's disease also published clinical criteria for the diagnosis of frontotemporal dementia in $2001 .^{4}$ Several other disorders present with frontotemporal symptoms, such as frontal lobe tumours, alcohol associated dementia, Huntington's disease, and Creutzfeldt-Jakob disease. Frontal lobe symptoms may also be found in other dementia disorders, such as Alzheimer's disease $(\mathrm{AD})$ and vascular dementia (VAD).

Interest in FTD has grown during the past decade, after it was found that hereditary FTD is associated with mutations in the tau gene. ${ }^{5}$ Furthermore, recent findings suggest that it may be more common than previously regarded. ${ }^{6}$ A frontal lobe syndrome (FLS) may be even more common as it often accompanies dementia disorders such as $\mathrm{AD}$ and VAD. Neuropathological studies of dementia have suggested that $10 \%$ of demented subjects suffer from $\mathrm{FTD},{ }^{78}$ while a recent clinical study reported a prevalence of $3.2 \%$ among patients evaluated at a memory clinic. ${ }^{9}$ Most previous studies on the prevalence of FTD are based on data from the health care system. ${ }^{10}{ }^{11}$ The two population studies published ${ }^{12}{ }^{13}$ included only cases of FTD who also fulfilled criteria for dementia according to DSM-III-R or short-CARE, but dementia as defined by DSM-III-R or similar criteria is not a requirement for the diagnosis of FTD in current criteria. FTD cases not fulfilling criteria for dementia may therefore have been missed in those latter studies. The reported prevalence of FTD is low, $0.015 \%$ before age $65^{10}{ }^{11}$ and $0-0.6 \%$ above age $65 . .^{12}$

No population study has reported on the prevalence of FLS or FTD by applying FTD criteria directly on unselected populations, and studies in very old individuals are lacking.

We aimed to study the prevalence of the FLS and fvFTD using the Lund-Manchester research criteria $(\text { LMRC) })^{2}$ in a representative sample of 85 year olds in Gothenburg, Sweden. ${ }^{14-18}$ The data in this epidemiological study include a wide range of psychiatric, neurological, and other variables, including the core diagnostic features of the LMRC. We were therefore able to design a symptom algorithm based on the LMRC, even though the data were collected before the LMRC were published. However, symptoms of language disturbances were not included in the algorithm, as these symptoms are unspecific in this age group and may be caused by a number of different disorders, including dementia and stroke. Furthermore, symptoms of language disturbance do not distinguish FTD from Alzheimer's disease (AD), as language disturbances are also common in $\mathrm{AD} .{ }^{19}$ Finally, although progressive aphasia

Abbreviations: $A D$, Alzheimer's disease; $C T$, computed tomography; $E E G$, electroencephalography; FLS, frontal lobe syndrome; FTD, frontotemporal dementia; fvFTD, frontal variant frontotemporal dementia; LMRC, Lund-Manchester research criteria; SPECT, single photon emission computed tomography; VAD, vascular dementia 


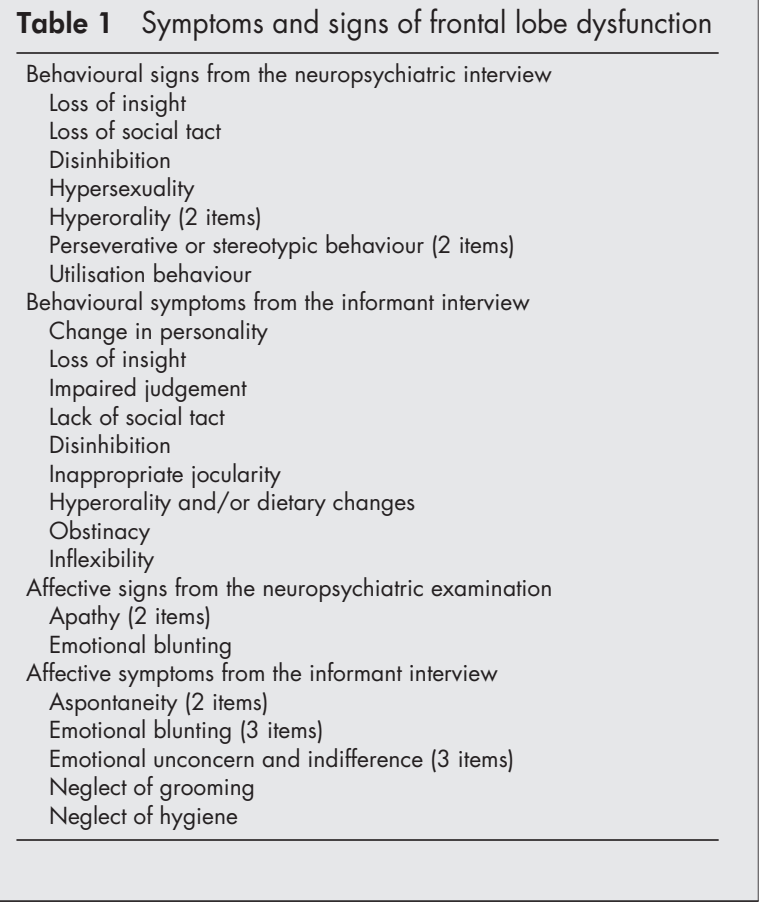

and semantic dementia are part of the FTD spectrum, the frontal variant accounts for the great majority of cases, ${ }^{6}$ and behavioural symptoms may occur in the other variants. ${ }^{4}$ Our study thus focuses on the frontal variant FTD, as described by Hodges and Miller, ${ }^{1}$ and is the first study to apply the LMRC directly on a representative population, living in the community.

\section{SUBJECTS AND METHODS}

\section{Subjects}

In 1986-87, all 85 year olds born between 1 July 1901 and 30 June 1902, registered for census purposes in Gothenburg, were invited to take part in a health survey; half of those were systematically selected for a psychiatric examination. A total of 494 individuals (response rate 63\%) agreed to participate in the neuropsychiatric examination $(\mathrm{n}=494 ; 143$ men, 351 women). Both people living in the community and those in institutions were included. This sample has been described in detail previously, and was found to be representative for the 85 year olds in Gothenburg with regard to sex, marital status, psychiatric registration, three year mortality rate, and institutionalisation. ${ }^{16}$ The mean age at the neuropsychiatric examination was 85 years 5 months (range 85 years 3 months to 86 years 1 month).

The Ethics Committee for Medical Research at Gothenburg University approved the study. Informed consent was obtained from the subjects, the nearest relatives, or both.

\section{Methods}

The neuropsychiatric examination was semistructured and performed by a trained psychiatrist in the subject's home or at institutions, and included ratings of symptoms and signs common in dementia and tests of mental functioning. ${ }^{16}$ Psychiatric symptoms and signs were rated with the Comprehensive Psychopathological Rating Scale..$^{20}$

A semistructured telephone interview with a close informant was performed by the psychiatrist for 451 individuals $(91 \%)$ and included questions on cognitive, emotional, and behavioural symptoms, and about age at onset and course of these symptoms. Among those who did not have a key informant interview, one was demented and 42 were non-demented. Only those who had both a psychiatric examination and a key informant interview were included in

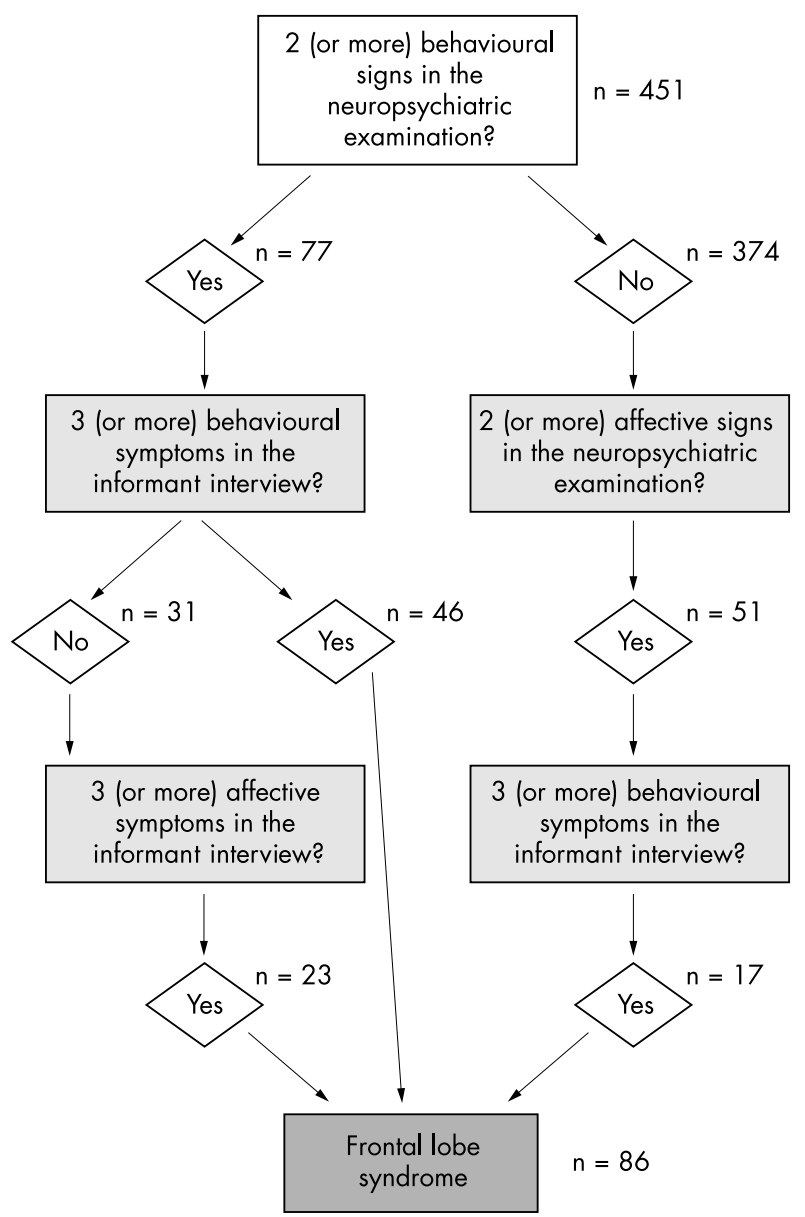

Figure 1 Algorithm for frontal lobe syndrome.

this paper ( $\mathrm{n}=451 ; 131$ men, 320 women). There were no significant differences between those with and without a key informant interview regarding relevant symptoms in the psychiatric examination. The data were collected blindly to any diagnostic aspects.

\section{Diagnostic procedures}

The Lund-Manchester research criteria (LMRC) ${ }^{2}$ include three frontotemporal dementia symptom constellations: (1) behavioural symptoms, (2) affective symptoms, and (3) speech disorder. The onset has to be insidious and the course invariably progressive. The criteria do not describe in detail the required severity of the symptoms, or how many symptoms or symptom constellations have to be present for a diagnosis. We therefore constructed an algorithm for the identification of FLS based on the core symptoms of FTD from the LMRC noted during the psychiatric examination and the close informant interview. The symptoms were selected to avoid misclassification with other disorders. Therefore, symptoms of language disturbance were not included, as language disturbance does not separate AD from FTD. ${ }^{19}$ For a symptom to be classified as present it had to lead to significant disturbance. The symptoms were grouped into four clusters: (1) behavioural signs typical for FTD from the neuropsychiatric examination, (2) behavioural symptoms typical for FTD from the informant interview, (3) affective symptoms typical for FTD from the neuropsychiatric examination, and (4) affective symptoms typical for FTD from the informant interview. The individual symptoms selected in the different symptom constellations were based on the description in the LMRC, and are shown in table 1.

Figure 1 presents the algorithm for the identification of FLS. An individual had to have a minimum number of 


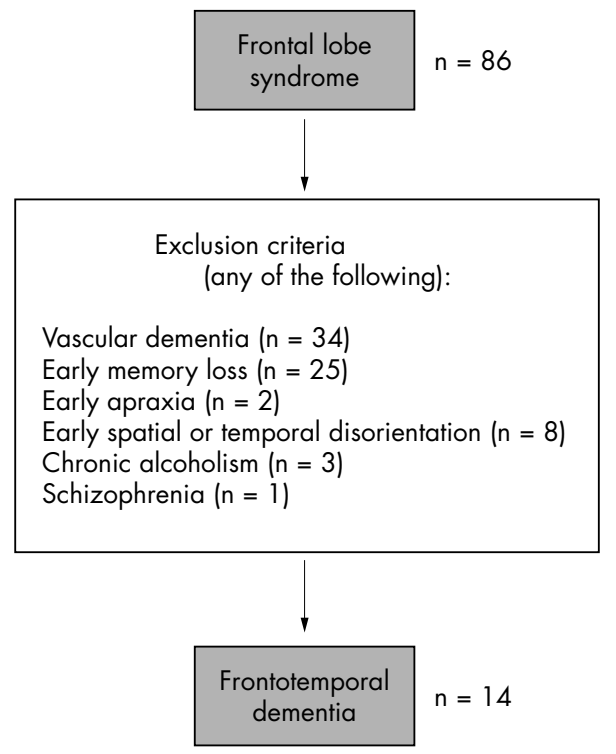

Figure 2 Exclusion criteria for frontotemporal dementia.

symptoms in both the neuropsychiatric examination and the informant interview, and always a minimum number of behavioural symptoms. Thus if an individual had two or more behavioural symptoms in the neuropsychiatric examination, he or she had to have at least three behavioural symptoms in the informant interview in order to be classified as having FLS. If there were less than two behavioural symptoms in the neuropsychiatric examination, it was required that at least two affective symptoms in the neuropsychiatric examination should be present and at least three behavioural symptoms in the informant interview. If an individual had two (or more) behavioural symptoms in the neuropsychiatric examination, but fewer than three behavioural symptoms in the informant interview, at least three affective symptoms had to be present in the informant interview for a classification of FLS (fig 1).

Frontal variant frontotemporal dementia was diagnosed in subjects with FLS who presented with only frontal lobe symptoms or with behavioural symptoms that clearly preceded (by two years or more) memory loss or other cognitive signs, such as agnosia or dyspraxia. The diagnosis of fvFTD could not be applied in the presence of early onset of memory problems, early spatial disorientation, early apraxia, vascular dementia, chronic alcoholism, and schizophrenia (exclusion criteria shown in fig 2).

The diagnosis of dementia was based on the neuropsychiatric examination and the close informant interview using the criteria of the Diagnostic and Statistical Manual of Mental Disorders, third edition-revised (DSM-III-R). ${ }^{21}$ The interobserver reliability for symptoms and signs and regarding causes of dementia has been reported previously and was found to be satisfactory. ${ }^{16}$ Individuals with dementia were classified into aetiological subgroups: Alzheimer's disease according to the criteria of the National Institute of Neurological and Communicative Disorders and Stroke and the Alzheimer's Disease and Related Disorders Association (NINCDS-ADRDA) $)^{22}$; and vascular dementia and dementia due to other causes as proposed by Erkinjuntti and colleagues. ${ }^{23}$ The aetiological diagnoses were based on all available information as described previously.

Dementia according to DSM-III-R was not a requirement for a diagnosis of FLS or fVFTD. The diagnoses of FLS and fvFTD were done blindly to diagnoses of dementia according to the DSM-III-R.

\section{Computed tomography (CT) of the brain}

All demented $(\mathrm{n}=145)$ and a systematic subsample of 269 non-demented individuals were invited to undergo CT
Table 2 The prevalence of frontal lobe syndrome and frontal variant frontotemporal dementia in 85 year olds

\begin{tabular}{lccl}
\hline & $\begin{array}{l}\text { Men } \\
(\mathrm{n}=131) \\
\mathrm{n}(\%)\end{array}$ & $\begin{array}{l}\text { Women } \\
(\mathrm{n}=320) \\
\mathrm{n}(\%)\end{array}$ & $\begin{array}{l}\text { Total } \\
(\mathrm{n}=451) \\
\mathrm{n}(\%)\end{array}$ \\
\hline $\begin{array}{l}\text { Frontal lobe syndrome } \\
\begin{array}{l}\text { Frontotemporal dementia } \\
\text { (frontal variant) }\end{array}\end{array}$ & $25(18.9)$ & $61(19.0)$ & $86(19.1)$ \\
\hline & $6(4.5)$ & $8(2.5)$ & $14(3.1)$ \\
\hline
\end{tabular}

scanning of the head. Of these, 103 demented $(71.0 \% ; 28$ men, 75 women) and 135 non-demented individuals (50.2\%; 46 men, 89 women) underwent the examination. As previously described, ${ }^{24}$ there were no significant differences between participants and non-participants within the demented and nondemented groups, with regard to any of the parameters studied (sex, marital status, registration as a psychiatric outpatient or inpatient, mental disorders, institutionalisation, three year mortality rate, history of focal neurological symptoms and signs, cardiovascular disorders, hypertension, or mean systolic and diastolic blood pressure).

All CT scans were performed without contrast enhancement and with $10 \mathrm{~mm}$ continuous slices on a Philips Tomoscan 310 or on a General Electric 8800. Two experienced radiologists, who were blind to the results of the neuropsychiatric examination, examined the CT scans. The occipital, parietal, frontal, and temporal lobes were categorised using a three point scale (normal, mild and moderate, or severe) according to the estimated extent of brain atrophy. ${ }^{25}$

\section{RESULTS}

\section{Prevalence of FLS}

Figure 1 shows the selection procedure for FLS. The prevalence of FLS was $19 \%(n=86)$, with no difference between men and women (table 2). Of those with FLS, 75 (87\%) were diagnosed with other types of dementia according to the DSM-III-R criteria (see table 3). Dementia according to the DSM-III-R was diagnosed in 145 (32.2\%) individuals; thus $52 \%(\mathrm{n}=75)$ of the demented fulfilled criteria for FLS, compared to $3.6 \%(\mathrm{n}=11)$ among the non-demented.

Among the 11 individuals who fulfilled criteria for FLS, but not for dementia according to DSM-III-R, nine were classified as fvFTD, one had early onset of apraxia, and one had a stroke at age 81 with a change in personal conduct and aphasia. These two latter cases were therefore not diagnosed as fvFTD.

\section{Prevalence of fvFTD}

Figure 2 shows the selection of cases for fvFTD. The prevalence of fvFTD was 3.1\% ( $n=14$; table 2$)$. Of those with fvFTD, $64 \%$

\begin{tabular}{|c|c|c|}
\hline \multicolumn{3}{|c|}{$\begin{array}{l}\text { Table } 3 \text { Number and proportion of } \\
\text { individuals with frontal lobe syndrome } \\
\text { in non-demented and demented } 85 \\
\text { year olds }\end{array}$} \\
\hline & \multicolumn{2}{|c|}{ FLS cases } \\
\hline & $n$ & $\%$ \\
\hline Dementia $(n=145)$ & 75 & 51.7 \\
\hline$A D(n=63)$ & 32 & 50.7 \\
\hline $\operatorname{VAD}(n=69)$ & 34 & 49.3 \\
\hline Other $(n=13)$ & 9 & 69.2 \\
\hline No dementia $(n=306)$ & 11 & 3.6 \\
\hline
\end{tabular}


$(n=9)$ did not fulfil criteria for dementia according to DSMIII-R, and five had previously been classified as AD.

\section{Frontal atrophy on computerised tomography}

CT scan was performed in 238 individuals, including 53 cases of FLS and six cases of fvFTD. Among those with FLS, 92.5\% $(\mathrm{n}=49)$ had moderate to severe frontal atrophy, compared to $48.6 \%(\mathrm{n}=90)$ of those without FLS. All six cases with fvFTD had moderate to severe frontal atrophy.

Among those with moderate to severe frontal atrophy $(\mathrm{n}=139), 49(35.3 \%)$ had FLS. Only four $(2.9 \%)$ among those without moderate to severe frontal atrophy had FLS.

\section{DISCUSSION}

Our study is the first to estimate the prevalence of the FLS and fvFTD in a very old population sample. We found that $3 \%$ in this population of 85 year olds were classified as having fvFTD according to the LMRC. Previous studies have reported much lower rates. A study in the Netherlands, based on referrals to specialist clinics or nursing homes, reported a prevalence of $0.003 \%$ in the age group $60-70$ years. ${ }^{26}$ Two recent English studies reported a prevalence of $0.015 \%$ in the age group below 65 years, one based on patients referred to specialist clinics, ${ }^{11}$ the other based on patients from general practice physicians and other health care professionals and institutions. ${ }^{10}$ Studies based on patient samples might heavily underestimate the prevalence of FTD.

Two population studies have so far reported on the prevalence of FTD in individuals above age 65. A study from Japan found no cases of FTD, ${ }^{12}$ and a study from Islington, UK, reported a prevalence of $0.6 \%{ }^{13}$ However, the Japanese study included only individuals who had dementia according to DSM-III-R in the screening process, and the Islington study used short-CARE to screen for dementia. In our study, nine of 14 fvFTD cases did not fulfil criteria for dementia, and would have been missed with such an approach, thus underestimating the prevalence of FTD. If the same proportion of FTD cases were non-demented in the Islington study as in our study, the prevalence of FTD in Islington would be around 2\%.

It is generally reported that FTD occurs mainly among individuals in the age group 50-70 years. ${ }^{27}{ }^{28}$ Our finding that 3\% of 85 year olds had fvFTD suggests that fvFTD may be more common in the very elderly than previously supposed. It is possible that some of those are wrongly diagnosed as having other forms of dementia or different psychiatric conditions. ${ }^{29} 30$

The prevalence of FLS (19\%) was much higher than that of FTD. One reason for this high prevalence is the high prevalence of dementia in this age group. FLS was found in $50 \%$ of those with $\mathrm{AD}$ and in $49 \%$ of those with VAD. These findings are in line with previous reports. ${ }^{31}{ }^{32}$ Among all 85 year olds with dementia or fvFTD, $9 \%$ received a diagnosis of fvFTD, which is similar to previous reports from dementia clinics. $^{33}$

Few studies have examined the relation between frontal atrophy on structural brain imaging and frontal symptoms. Patients with FTD do not always show frontal atrophy on brain imaging in clinical series. ${ }^{29}{ }^{34}$ We found that $93 \%$ of those with FLS, and all cases with fvFTD, had moderate to severe frontal atrophy, which supports the value of our diagnostic algorithm. However, moderate to severe frontal atrophy was also found in $49 \%$ of those without FLS, showing that frontal atrophy is not specific for FLS, but can be seen in many conditions, including normal ageing and AD. Among those with moderate to severe frontal atrophy, only $35 \%$ had FLS, supporting the notion from others that frontal atrophy is not always related to frontal symptoms. ${ }^{35}$ These findings may also reflect the strict criteria for FLS in our study. We found that only 3\% of individuals without frontal atrophy were classified as FLS according to our symptom algorithm, supporting the validity of this approach.
Some limitations of the study have to be considered. First, our criteria were rather strict, requiring a large number of symptoms to be present for a diagnosis. This was done as we wanted to avoid overinclusion of cases, but we might therefore have missed some cases of FLS and fvFTD. This may be one reason why only a minority of those with moderate to severe frontal atrophy on CT had FLS. Second, symptoms of language disturbance were not included. This might also lead to underdiagnosis. The reason for not using language disturbance in the diagnosis is that these symptoms are unspecific and do not adequately separate AD from FTD.$^{19}$ Instead, we emphasised behavioural symptoms typical for FLS in the diagnosis. Third, response rate was $63 \%$. It is possible that persons with FLS declined to participate more often than other 85 year olds because of their behavioural symptoms. However, responders and non-responders were similar regarding a number of studied background factors. On the other hand, we excluded 43 subjects without a key informant interview. Almost all of those were non-demented, which might have overestimated the frequency of FLS in our population. Fourth, we used CT to evaluate frontal atrophy, whereas MRI would now be the preferred imaging investigation in patients with suspected FTD. Fifth, other examinations, such as single photon emission computed tomography (SPECT) and electroencephalography (EEG), were not available. The sensitivity of a hypofrontal perfusion pattern using CBF-SPECT is probably high, but the specificity rather low for FTD, ${ }^{36}$ and the use of EEG alone is most probably unspecific. ${ }^{37}$ Hence, it is not obvious that SPECT and EEG would have provided additional diagnostic information. Sixth, we could not confirm our diagnoses at postmortem examination as neuropathological investigations were not available, which is the case in most population studies. However, it has been suggested that a reliable diagnosis of FTD is possible on the basis of clinical symptoms and signs. ${ }^{28}$ We can, as in other studies relying on clinical examinations, not exclude the possibility that some of our FTD cases might have had the frontal variant of AD. This diagnosis is supposed to be rare $^{38}$ and can only be obtained at postmortem examination. Seventh, mild frontal lobe features have been suggested to be common in the elderly population as a reflection of a normal age associated cognitive decline. ${ }^{39}{ }^{40}$ However, the FLS diagnosed in this study is more advanced than what is expected as a normal age related change.

Finally, the data were collected seven years before the LMRC were published. This was possible as the epidemiological studies in Gothenburg include a wide range of psychiatric, neurological, and other variables, including the core diagnostic features of the LMRC. Although it is possible that the same patients had not been diagnosed if we had used a prospective or contemporary design, it is more likely that additional cases of FLS and FTD would have been diagnosed with a prospective design due to the severity demanded by the algorithm for a diagnosis. The possibility of circularity in the diagnosis was minimised, as the variables in the LMRC were collected blindly to any diagnostic aspects, and as the diagnoses of FLS and FTD were done blindly to diagnoses of dementia according to the DSM-III-R.

We found that FLS was common among 85 year olds, especially among those with dementia disorders. The prevalence of fvFTD was $3 \%$, which is considerably higher than expected in this age group. Our study also shows that a majority of those with fvFTD according to the LMRC do not fulfil DSM-III-R criteria for dementia.

\section{ACKNOWLEDGEMENTS}

The study is a project within the gerontological and geriatric population studies in Gothenburg, Sweden. Project leader 1971-87: Professor Alvar Svanborg; from 1988: Professor Bertil Steen. We thank Ms Liselott Ågren and Yvonne Sundin for technical assistance. The study was supported by grants from the Swedish Research Council (11267), 
the Swedish Council for Working Life and Social Research (0914), Stiftelsen Söderström-Königska Sjukhemmet, Konung Gustaf V:s och Drottning Victorias Stiftelse, Stiftelsen för Gamla Tjänarinnor, Handlanden Hjalmar Svenssons Forskningsfond, and Stiftelsen Professor Bror Gadelius' Minnesfond.

\section{Authors' affiliations}

T B Gislason, M Sjögren, L Larsson, I Skoog, The Institute of Clinical Neuroscience, Department of Psychiatry, Sahlgrenska Hospital, Gothenburg, Sweden

\section{Competing interests: None declared}

\section{REFERENCES}

1 Hodges JR, Miller B. The classification, genetics and neuropathology of frontotemporal dementia. Introduction to the Special Topics Papers: Part 1. Neurocase $2001 ; 7: 31-5$

2 Brun A, Englund B, Gustafsson L, et al. Clinical and neuropathological criteria for frontotemporal dementia. I Neurol Neurosurg Psychiatry 1994;57:416-18.

3 Neary D, Snowden JS, Gustafsson L, et al. Frontotemporal lobar degeneration. A consensus on clinical diagnostic criteria. Neurology 1998:51:1546-54

4 McKhann GM, Albert MS, Grossman M, et al. Clinical and pathological diagnosis of frontotemporal dementia. Report of the work group on frontotemporal dementia and Pick's disease. Arch Neurol 2001;58:1803-9.

5 Wilhelmsen K, Clark LN, Miller BL, et al. Tau mutations in frontotemporal dementia. Dement Geriatr Cogn Disord 1999;10(suppl 1):88-92.

6 Snowden JS, Neary D, Mann DMA. Frontotemporal dementia. Br J Psychiatry 2002;180:140-3.

7 Brun A, Gustafsson L. The longitudinal dementia study: a 25-year perspective on neuropathology, differential diagnosis and treatment. In: Corain B, lqbal K, Nicolini M, et al, eds. Alzheimer's disease: advances in clinical and basic research. Chichester: John Wiley and Sons Ltd, 1993.

8 Neary D, Snowden JS. Fronto-temporal dementia: nosology, neuropsychology, and neuropathology. Brain Cogn 1996;31:176-87.

9 Andreasen N, Blennow K, Sjödin C, et al. Prevalence and incidence of clinically diagnosed memory impairments in a geographically defined general population in Sweden. Neuroepidemiology 1999;18:144-55.

10 Harvey RJ, Rossor MN, Skelton-Robinson M, et al. Young onset dementia: epidemiology, clinical symptoms, family burden, support and outcome, 1998. Available at: www.dementia.ion.ac.uk.

11 Ratnavalli E, Brayne C, Dawson K, et al. The prevalence of frontotemporal dementia. Neurology 2002;58:1615-21.

12 Yamada T, Hattori H, Miura A, et al. Prevalence of Alzheimer's disease, vascular dementia and dementia with Lewy bodies in a Japanese population. Psychiatry Clin Neurosci 2001;55:21-5

13 Stevens T, Livingston $G$, Kitchen $G$, et al. Islington study of dementia subtypes in the community. Br J Psychiatry 2002;180:270-6.

14 Rinder L, Roupe S, Steen B, et al. Seventy-year-old people in Gothenburg. A population study in a industrialized Swedish city. Acta Med Scand 1975;198:397-407.

15 Skoog I, Lernfelt B, Landahl S, et al. 15-year longitudinal study of blood pressure and dementia. Lancet 1996;347:1141-5.

16 Skoog I, Nilsson L, Palmertz B, et al. A population-based study of dementia in 85-year-olds. N Engl J Med 1993;328:153-8.

17 Steen B, Djurfeldt $\mathrm{H}$. The gerontological and geriatric population studies in Gothenburg, Sweden. Z Gerontol 1993;26:163-9.

18 Svanborg A. Seventy-year-old people in Gothenburg. A population study in a industrialized Swedish city. II. General presentation of social and medical conditions. Acta Med Scand 1977; (suppl 61 1):3-37.
19 Rosen HJ, Hartikainen KM, Jagust W, et al. Utility of clinical criteria in differentiating frontotemporal lobar degeneration (FTLD) from AD. Neurology 2002;58:1608-15.

20 Åsberg M, Schalling D. Construction of a new psychiatric rating instrument, the Comprehensive Psychopathological Rating Scale (CPRS). Prog Neuropsychopharmacol 1979;3:405-12.

21 American Psychiatric Association. Diagnostic and statistical manual of mental disorders-revised (DSMIII-r), 3rd edn. Washington, DC: APA, 1987.

22 McKhann G, Drachman D, Folstein M, et al. Clinical diagnosis of Alzheimer's disease: report of the NINCDS-ADRDA work group under the auspices of Department of Health and Human Services task force on Alzheimer's disease. Neurology 1984;34:939-44.

23 Erkinjuntti T, Haltia M, Palo J, et al. Accuracy of the clinical diagnosis of vascular dementia: a prospective clinical and post-mortem neuropathological study. J Neurol Neurosurg Psychiatry 1988;51:1037-44.

24 Skoog I, Palmertz B, Andreasson LA. The prevalence of white matter lesions on computed tomography of the brain in demented and non-demented 85-year-olds. J Geriatr Psychiatry Neurol 1994;7:169-75.

25 De Leon MJ, Ferris SH, George AE, et al. Computed tomography evaluations of brain-behaviour relationships in senile dementia of the Alzheimer's type. Neurobiol Aging 1980;1:69-79.

26 Stevens $M$, van Dujin CM, Kamphorst W, et al. Familial aggregation in frontotemporal dementia. Neurology 1998;50:1541-5.

27 Neary D, Snowden JS, Northen B, et al. Dementia of frontal lobe type. J Neurol Neurosurg Psychiatry 1988;51:353-61.

28 Gustafsson L. Clinical picture of frontal lobe degeneration of non-Alzheimer type. Dementia 1993;4:143-8.

29 Pasquier F, Lebert F, Lavenu I, et al. The clinical picture of frontotemporal dementia: diagnosis and follow-up. Dement Geriatr Cogn Disord 1999;10(suppl 1):10-14.

30 Varma AR, Snowden JS, Lloyd JJ, et al. Evaluation of the NINCDS-ADRDA criteria in the differentiation of Alzheimer's disease and frontotemporal dementia. J Neurol Neurosurg Psychiatry 1999:66:184-8.

31 Ishii N, Nishihara Y, Imamura T. Why do frontal lobe symptoms predominate in vascular dementia with lacunes? Neurology 1986:36:340-5.

32 Wallin A, Blennow K, Gottfries CG. Subcortical symptoms predominate in vascular dementia. Int J Geriatr Psychiatry 1991;6:137-45.

33 Tissot R, Constantinides J, Richard J. Pick's disease. In: Bruyn V, ed. Handbook of clinical neurology. Amsterdam: North-Holland Publishing Company, 1985:233-46

34 Scheltens $\mathbf{P}$, van Swieten JC. Neuroimaging in frontotemporal dementia. In: Pasquier $F$, Lebert $F$, Scheltens $P$, eds. Frontotemporal dementia. Dordrecht: ICG Publications, 1996:83-90.

35 Uterga Valiente JM, Rodriguez Iriarte ML, Gonzalez Garcia Al, et al. Demencia con atrofia frontal: estudio clinico de 18 pacientes con deterioro intelectual y degeneracion de los lobulos frontales segun la neuroimagen. Rev Neurol 2002;34:709-14.

36 Risberg J, Gustafson L. Regional cerebral blood flow measurements in the clinical evaluation of demented patients. Dement Geriatr Cogn Disord 1997;8:92-7

37 Julin $\mathbf{P}$, Wahlund LO, Basun $\mathrm{H}$, et al. Clinical diagnosis of frontal lobe dementia and Alzheimer's disease: relation to cerebral perfusion, brain atrophy and electroencephalography. Dementia 1995;6:142-7.

38 Brun A. Frontal lobe degeneration of non-Alzheimer type revisited. Dementia 1993:4:126-31.

39 Mittenberg W, Seidenberg M, O'Leary DS, et al. Changes in cerebral functioning associated with normal aging. J Clin Exp Neuropsychol 1989;1 1:918-32.

40 Parkin AJ, Lawrence A. A dissociation in the relation between memory tasks and frontal lobe tests in the normal elderly. Neuropsychologia 1994;32:1523-32. 